\title{
BotTLENECK DETECTION Algorithm To ENHANCE LIFETIME OF WSN
}

\author{
Vinaya Yarate $^{1}$ and Harish Kenchannavar ${ }^{2}$ and Umesh Kulkarni ${ }^{3}$ \\ ${ }^{1}$ Vinaya V. Yarate, M Tech Student CSE, Gogte Institute of Technology, Belgaum, India. \\ ${ }^{2}$ Harish H. Kenchannavar, Dept of Computer Science and Engineering, KLS Gogte \\ Institute of Technology, Belgaum, India. \\ ${ }^{3}$ Umesh M. Kulkarni, Dept of Computer Science and Engineering, KLS Gogte Institute \\ of Technology, Belgaum, India.
}

\begin{abstract}
In recent years, a wireless sensor network is gaining much more importance due to its immense contribution in numerous applications. Deployment of sensor nodes that would reduce computation, minimize cost and gaining high degree of network connectivity is an challenging task. Random deployment of sensor nodes causes the wireless sensor networks to face topological weaknesses such as communication bottlenecks, network partitions and sensing holes. These problems lead to uneven energy utilization, reduction in reliability of network and reduction in network lifetime. Bottleneck detection algorithm is proposed to identify bottleneck and minimal bottleneck zones in network. Additional sensor node deployment strategy is used in bottleneck detection algorithm to extend network lifetime. Random additional sensor node deployment and Targeted additional sensor node deployment are proposed to enhance network lifetime. Deployment strategies are compared with respect to network parameters such as throughput, packet delivery fraction and network lifetime.
\end{abstract}

\section{KEYWORDS}

Wireless sensor networks, Node Deployment, Random Additional Senor deployment, Targeted Additional Senor Deployment, Energy efficiency.

\section{INTRODUCTION}

Recent years have witnessed an increased interest in the use of wireless sensor networks (WSNs) in numerous applications such as forest monitoring, disaster management, space exploration, and battlefield surveillance. Sensor nodes are characterized by possessing limited battery that have small amount of energy. Sensor nodes are usually deployed in defected regions which causes recharge or replacement of the battery difficult. Therefore, in WSNs minimizing energy utilization of every sensor node in given network topology is one of the important issues to be considered in enhancing network lifetime.

Sensor nodes are deployed in a sensing field to gather essential information from it. It is infeasible to locate sensor positions and network topology before the deployment. Therefore, the deployed sensors must self-organize to set up barriers in distributed manner. In wireless sensor networks, failure of sensor nodes below a pre-determined level causes the network to fall below a desired level of "quality" and we must know which parts of network need to be repaired. These weakest set of nodes in given network topology is called as bottleneck zone. In bottleneck zones, sensor nodes vacate their energy quickly and results in creating energy hole problem in WSN. 
Thus, bottleneck zone needs to be identified and additional sensor nodes must be deployed in order to prolong network lifetime.

In this paper, an algorithm called bottleneck finding is proposed to identify weakest areas in wireless sensor networks. In section 2, previous works related to enhancing lifetime of network is summarized. In section 3, deployment methodologies are discussed. In section 4, Bottleneck detection algorithm is proposed. In section 5, simulation results are discussed.

\section{RELATED WORK}

In [2], authors formulated study on barrier coverage issue and addressed a solution for it. An algorithm called, PUSH-PULL-IMPROVE is proposed in this paper, which focuses on study of minimum-cost barrier coverage issue and formulated a solution for it in asynchronous wireless sensor networks. Proposed algorithm can also be studied with respect to local barrier coverage. In [3], authors have addressed k-barrier coverage problems, minimum cost k-barrier coverage problem, etc. Simulation results shown in this paper depict that proposed algorithms are much more efficient. In paper [1], applications of sensor networks are addressed. Based on requirements of services and functionality, an layered communication architecture is designed. To enhance features of layered architecture, various energy efficient routing protocols and algorithms are designed. Various performance metrics such as flexibility of sensor nodes, deployment capability, cost, etc. are discussed. In [4], authors presented relation between energy consumption and deployment strategy. Comparision among random and grid deployment strategies is carried based on energy utilization. In [7], authors presented study on k-discrete barrier coverage. Various algorithms such as Sleeping Schedule (G-KSS), KLCML, and Redeployment algorithms are proposed. In [8], authors formulated study on extending the lifespan of event-driven sensor networks. In [19], study on weak barrier coverage is presented. In [10], authors have discussed the fault of sleep-scheduling technique and also addressed joint-routing technique. Iterative Geometric Programming (IGP) technique is used to address signomial program (SP) problem. Various deployment strategies such as hybrid strategy, connectivityoriented strategy and lifetime-oriented strategy are addressed in [13]. The concepts of minimal bottleneck zones, deployment of additional sensor nodes in weak-prone areas and immobile sensors are addressed in this research paper. In [26], authors proposed study on network connectivity, network lifetime and coverage problem. In [27], authors addressed coverage redundancy problem. An lightweight deployment-aware scheduling algorithm is proposed. In [6], authors presented a study on multi-round sensor node deployment strategy. Study with respect to 1-barrier coverage is only carried in this paper. In [23], authors presented study on deployment platform called POWER, which focuses on minimizing deployment cost and maximizing efficiency of sensor node deployment. In [25], authors presented study on coverage and connectivity problems. Different deployment strategies such as square based deployment and equilateral triangle deployment strategy is proposed in this paper. In [11], study on area coverage with respect to different deployment strategies is carried.

\section{NETWORK MODEL}

\subsection{Existing System}

In wireless sensor network, wireless communication protocols efficiency need to be enhanced to prolong battery lifetime of wireless devices [8]. Sensors in network area are energy constrained. Thus, sensors in network frequently wakes up to sense the channel to forward the data. Each time a sensor node senses the channel, a small portion of energy is utilized without making the transmission. After some duration, sensor nodes energy gradually decreases resulting in less 
transmission of packets and nodes spend most of their lifetime in sleeping mode. Thus, a particular wireless sensor network is structurally damaged and needed to be repaired. These weakest set of nodes in given network topology is called as minimal bottleneck and bottleneck zones. Minimal bottleneck nodes are the nodes with least energy and are about to vanish from the network. Bottleneck nodes are the nodes in which they have no energy to transmit the data and thus are considered as dead. Sensor nodes in minimal bottleneck and bottleneck regions vacate their energy quickly. This results in creation of energy hole problem in wireless sensor network. Thus, minimal bottleneck and bottleneck zones needs to be identified and additional sensor nodes must be deployed in weakest areas in order to prolong network lifetime.

\section{Limitations of existing system}

Due to continuous energy level degradation in the existing system, it leads to network isolation, frequent break up of links, and network lifetime goes on decreasing.

\subsection{Proposed System}

The proposed system is based on identification of minimal bottleneck and bottleneck regions in network topology. Sensor nodes are equipped with minimum battery power and thus, increasing the lifetime of wireless sensor networks becomes crucial. To enhance lifetime of sensor nodes, energy efficient routing protocols must be improved which minimizes the maintenance cost and maximizes the overall performance of the nodes. Energy utilization is maximum in transmission and reception of data packets. After deployment of sensor nodes, it is infeasible to recharge the batteries. Thus energy must be conserved and properly balanced among all the sensors in network.

At the initial stage, sensor nodes forward huge amount of data to the sink. After some duration, due to energy level variations in the network, lifetime gradually decreases. For these purpose, two deployment strategies are proposed : Random and Targeted. Random extra sensor node placement prolongs network lifetime by placing extra sensor nodes everywhere in complete thin belt area. Targeted extra sensor node placement prolongs lifetime of network by placing extra sensor nodes everywhere in minimal bottlenecks area arbitarily. Deployment region is different for both the strategies. These additional node deployment strategies utilizes bottleneck functions to prolong network lifespan. As soon as energy in sensor nodes falls below a pre-determined level, those sensor nodes are unable to transmit the data to the sink, resulting into frequent break up of links, and leads to reduction in network lifetime. Thus, minimal bottleneck and bottleneck zones are identified based on energy levels in sensor nodes and additional sensors are deployed in those areas in order to prolong the network lifetime.

\section{Advantages of Proposed System}

As minimal bottleneck and bottleneck regions are detected by algorithm in the network, the weak areas are replaced by additional sensor node deployment strategies. Thus by random additional and targeted additional deployment strategies in the proposed work, it facilitates formation of stable links in the network by enhancing network lifetime. 


\subsection{Additional Sensor Node Deployment}

\section{Case 1: Random Additional Sensor Node Deployment}

In this deployment strategy, sensor nodes are randomly deployed in two regions : thin region and entire region. By minimal bottleneck finding algorithm, bottleneck zones are detected in the network. In thin belt region, bottleneck nodes are detected in some portion of network and additional random deployment is done in that area. In entire region, bottleneck nodes are detected in the entire network area and additional deployment is done throughout the network. Thus, random additional sensor deployment is done with respect to two regions : thin region and entire region, and parameters like overall network lifetime, throughput, packet delivery fraction are computed.

\section{Case 2: Targeted additional Sensor Deployment}

In this deployment strategy, bottleneck sensor nodes are targeted and additional senor deployment is done with respect to those targeted nodes. Parameters like network lifetime, throughput, and packet delivery fraction are computed with respect to targeted nodes.

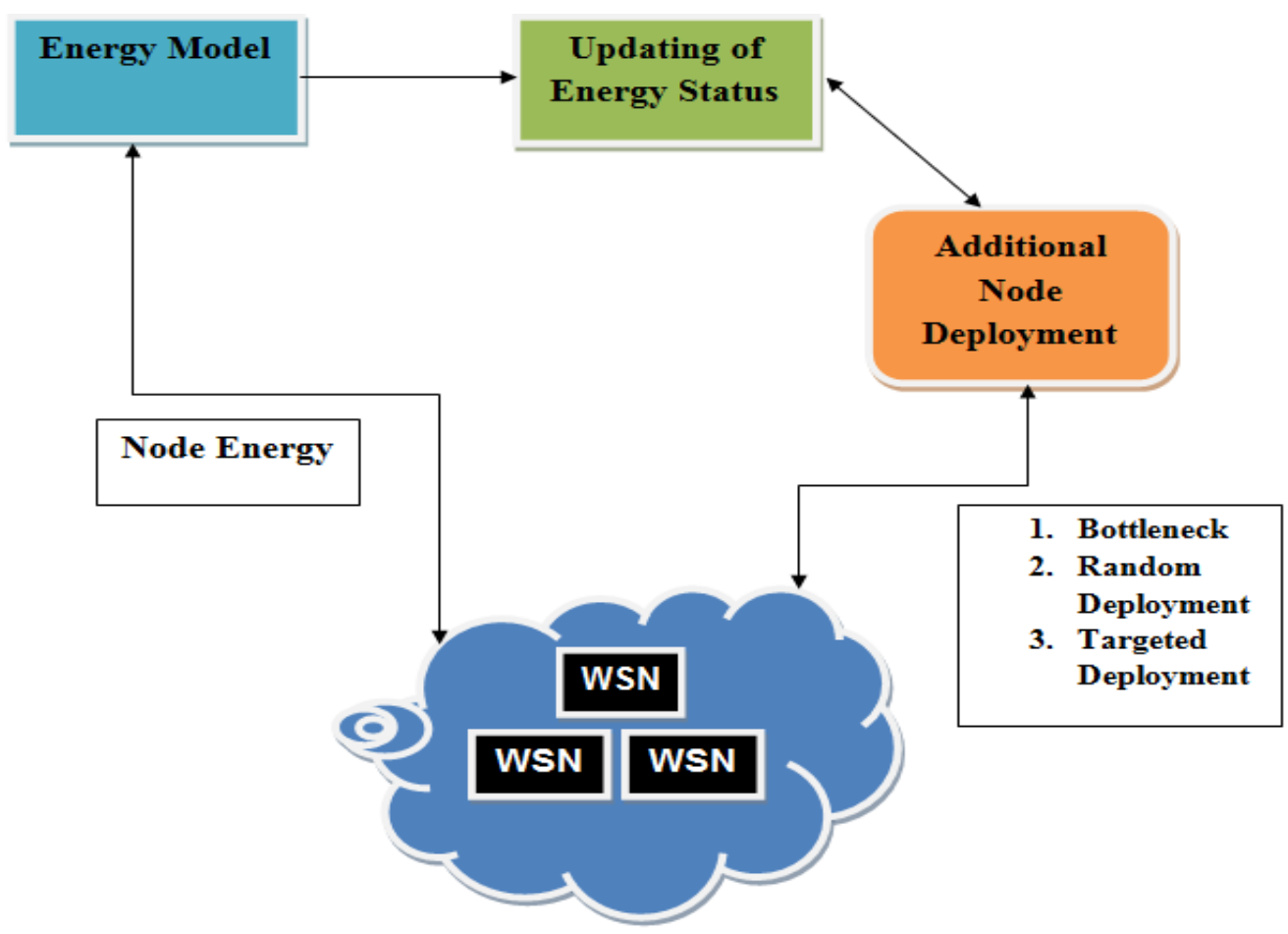

Figure 1. Flow Diagram

Figure 1. Depicts, flow diagram of proposed system. It consists of energy model, updating of energy status, and node replacement. In energy model, energy levels such as initial energy, consumed energy, remaining energy of every sensor nodes is computed. In updating of energy status, nodes energy are updated. Due to energy level variations in wireless sensor networks, it results into minimal bottleneck and bottleneck zones. These zones are replaced with random additional nodes and targeted additional nodes, to enhance network lifetime. 
International Journal of Next-Generation Networks (IJNGN) Vol.8, No.2/3, September 2016

\subsection{Properties of Bottleneck Detection Algorithm relating to Wireless Sensor Networks}

- Initially, all sensors in network area are immobile in nature and are energy constrained.

- Every sensor node operates sensing tasks at regular intervals and diffuse the information directly to sink.

- Multi-hop routing method is used by every sensor nodes in the network to forward the data to the closest relay node.

- Sink node has capacity to interact with every sensor nodes in network [11].

- Every node is assigned with a unique number based on their location in the network.

- As per the bottleneck detection algorithm, weakest zones in the network are identified and must be repaired to enhance network lifetime by deploying additional sensors in those weak-prone areas.

- Minimal bottleneck nodes are the nodes with least energy and are about to vanish from the network.

- Bottleneck nodes are the nodes in which they have no energy to transmit the data and thus are considered as dead.

- Minimal bottleneck and bottleneck nodes have the ability to vacate their energy quickly.

\section{BOTTLENECK DETECTION ALgORITHM}

Input: Set of sensor nodes $S$, Energy levels : $\mathrm{E}_{\text {init, }} \mathrm{E}_{\text {resd, }} \mathrm{E}_{\text {cons. }}$ Output: Set of bottleneck nodes.

Step 1: Assume a wsn in which a massive number of small battery-driven senor nodes are randomly deployed in target fields, say,

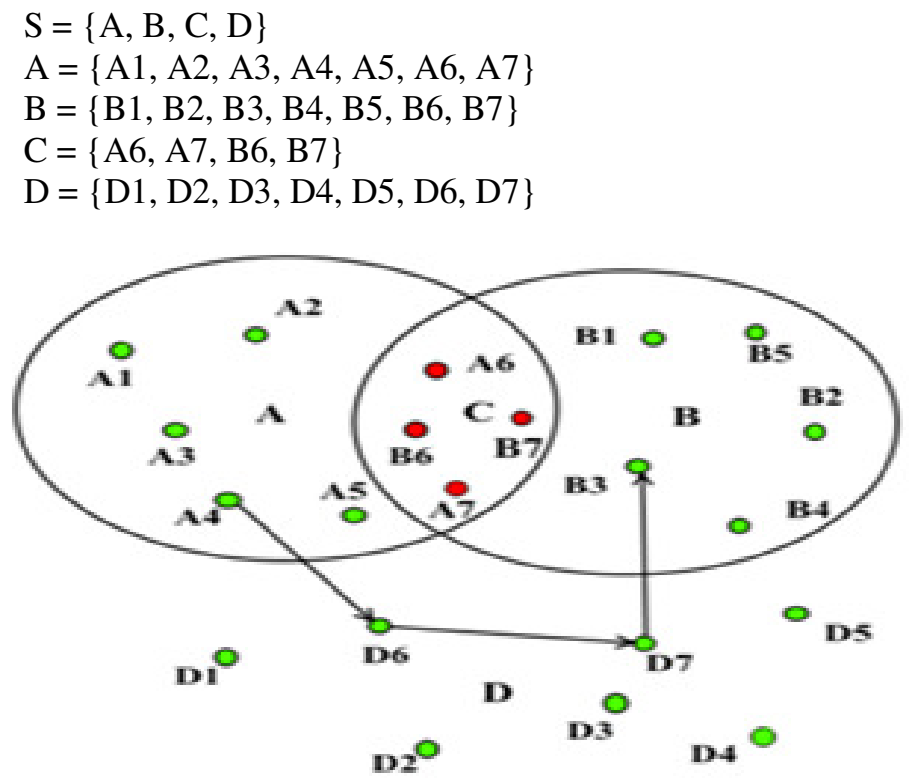

Figure 2. Bottleneck Region Detection

Step 2: After the initial deployment, all sensor nodes communicate with neighboring nodes that are within their sensing range by distance formula: 


$$
d\left(S_{i} S_{i}\right)=\sqrt{\left(X_{i}-X_{i}\right)^{2}+\left(Y_{i}-Y_{i}\right)^{2}}
$$

Where, " $\mathrm{S}_{\mathrm{i}}$ " and " $\mathrm{Sj}$ ” are any two sensors located at positions $\left(\mathrm{X}_{\mathrm{i}}, \mathrm{X}_{\mathrm{j}}\right)$ and $\left(\mathrm{Y}_{\mathrm{i}}, \mathrm{Y}_{\mathrm{j}}\right)$.

Step 3: 1 . Calculate energy levels: $E_{\text {init, }} E_{\text {resd, }} E_{\text {cons }}$ in each target fields.

2. $\mathrm{E}_{\text {resd }}=\mathrm{E}_{\text {init }}-\mathrm{E}_{\text {resd }}$ Where, $\mathrm{E}_{\text {init }}=$ Initial energy

$\mathrm{E}_{\mathrm{red}}=$ Residual energy

$\mathrm{E}_{\text {cons }}=$ Consumed energy

3. Lifetime of network:

$$
\text { Average Energy }=\frac{\text { Total Enaergy }}{\text { No, of nodes }(\mathrm{n})}
$$

Step 4: Assume target field ' $\mathrm{C}$ ' as set of candidate nodes.

1. Sensor nodes in field $C$ notifies all its neighbors in area $A\{A 1, A 2, A 3, A 4, A 5, A 6\}$ and $B$ $\{\mathrm{B} 1, \mathrm{~B} 2, \mathrm{~B} 3, \mathrm{~B} 4, \mathrm{~B} 5, \mathrm{~B} 6\}$.

2. Neighbour nodes will find other possible ways to link different partitions such as A-D-C.

3. Select any one random node in area A to deliver a message for confirmation to any arbitrary node in region $\mathrm{B}$.

4. Within a determinate time of threshold $\mathrm{T}$, if the sensor node in the given area $\mathrm{B}$ gets the message from any arbitrary node in area $\mathrm{A}$, then nodes from target field $\mathrm{C}$ cannot be bottleneck nodes $\left(\mathrm{E}_{\mathrm{resd}} \neq 0\right)$.

5. However, if it does not receive message, nodes from target field $\mathrm{C}\{\mathrm{A} 6, \mathrm{~A} 7, \mathrm{~B} 6, \mathrm{~B} 7\}$ is a set of bottleneck nodes $\left(\mathrm{E}_{\mathrm{red}}=0\right)$.

Step 5: End.

\section{Simulation RESUltS}

The performance of Bottleneck Detection Algorithm is evaluated using NS 2.35 simulator. The simulation work is carried out in NS2.35 simulator for the effect of additional sensor nodes deployed on lifetime.

\subsection{NAM Window}

Network Console window Executes NAM Visualization Window, which help for visualizing node placement and packet transmission and queue type. Packet Transmission in NAM Window are visualized as shown below. 


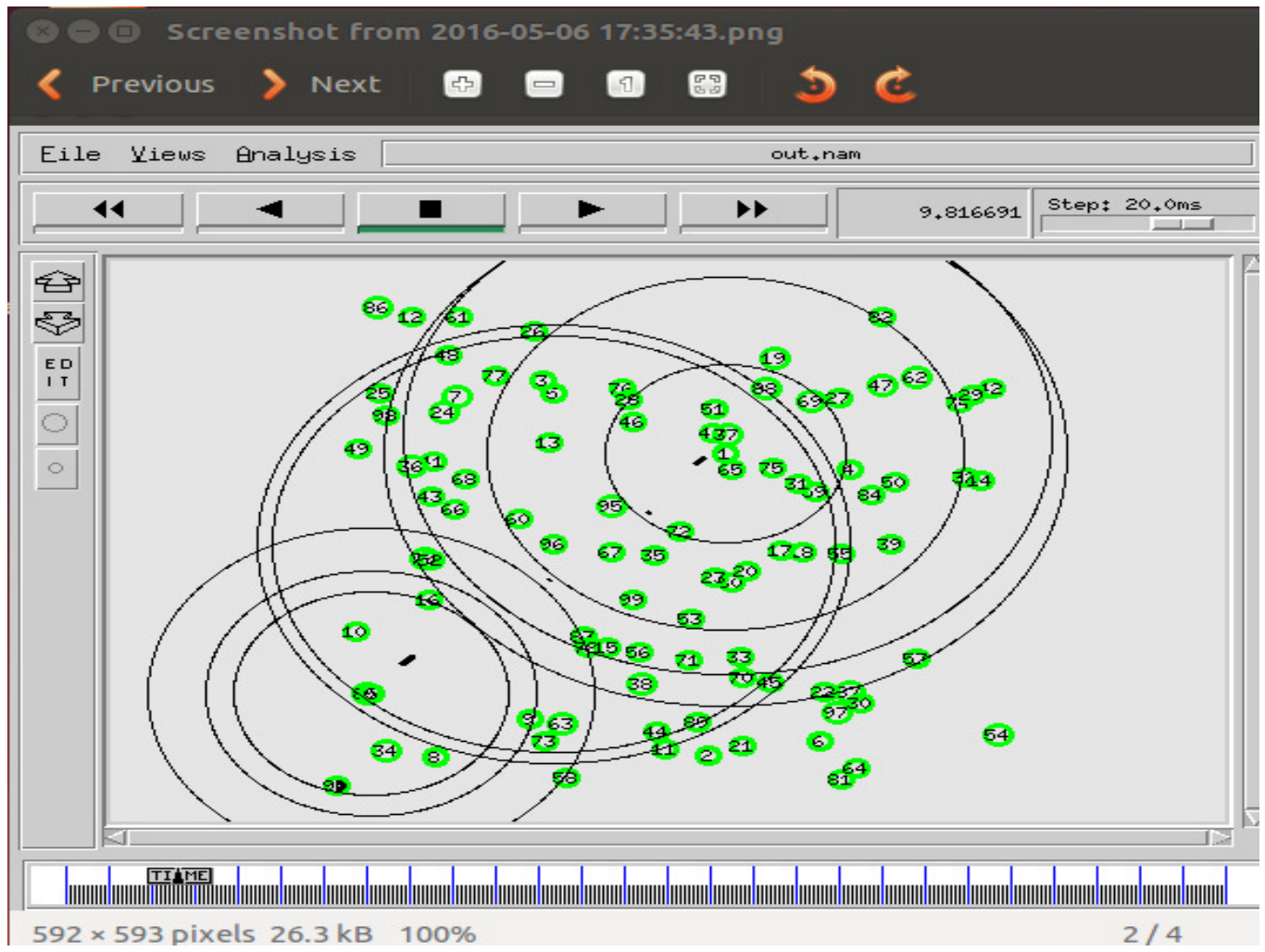

Figure 3 Shows NAM Window

\subsection{Radio Propagation Model}

As compared to wired channels, analysis of radio channels is more complex [31]. Radio propagation models are utilized to predict the received signal power of each packet. There are three radio propagation systems called shadowing system, two-ray ground reflection system, and free space system. In proposed work, two-ray ground reflection system is used.

\section{Two-ray ground reflection system}

Direct route and ground reflection route are taken into consideration in two-ray ground reflection model. As correlated to free space system, these system yields more authentic prediction at large distance. At distance d, power received is given by,

$$
P_{r}(d)=\frac{P_{t} G_{t} h_{t}^{2} h_{r}^{2}}{d^{4} L}
$$

Where, $\mathrm{P}_{\mathrm{t}}=$ signal power transmitted.

$\mathrm{G}_{\mathrm{r}}$ and $\mathrm{G}_{\mathrm{t}}=$ gains of antenna

$\mathrm{h}_{\mathrm{r}}$ and $\mathrm{h}_{\mathrm{t}}=$ receive and transmit antennas heights.

$\mathrm{L}=$ System loss.

Due to oscillation caused by combination of destructive and constructive rays, it does not yield good result for a short distance. 


\subsection{Energy Model}

Energy model shows level of energy in mobile host[32]. It is an node attribute. It consists of three parameters : initial energy (InitialEnergy), reception power (rxPower), transmission power (txPower). It maintains the total energy of network and does not maintain radio states. Analysis of energy levels is done through trace files. If the energy in node falls to zero, it indicates as dead node. Energy utilization of node is due to reception and transmission of data packets. To evaluate chunk of energy utilized during delivery process, txPower must be multiplied by time required to deliver a packet. To obtain nodes energy or energy model related information, energy model must be included in tcl file.

$$
\text { txEnergy }=\text { txPower } * \text { (packetsize/bandwidth })
$$

For received data packet

$$
\text { rxEnergy }=\text { rxPower } *(\text { packetsize } / \text { bandwidth })
$$

\begin{tabular}{|c|c|}
\hline Parameters & Values \\
\hline Network Interface & WirelessPhy \\
\hline MAC Type & WirelessChannel \\
\hline Channel & TwoRayGround \\
\hline Propagation & OmniAntenna \\
\hline Antenna & DropTail/PriQueue \\
\hline Queue & Energy in joules \\
\hline Initial energy & Receiving power in watts \\
\hline Reception Power & Transmitting power in watts \\
\hline Transmission Power & Power consumption in watts in sleep state \\
\hline Sleep Power & $\begin{array}{c}\text { Power consumption in state transition from } \\
\text { sleep to idle }\end{array}$ \\
\hline Transition Power & Time in seconds used in transition from sleep to \\
\hline Transition Time & \\
\hline
\end{tabular}

Table 1. Parameters of Energy Model in NS2 
International Journal of Next-Generation Networks (IJNGN) Vol.8, No.2/3, September 2016

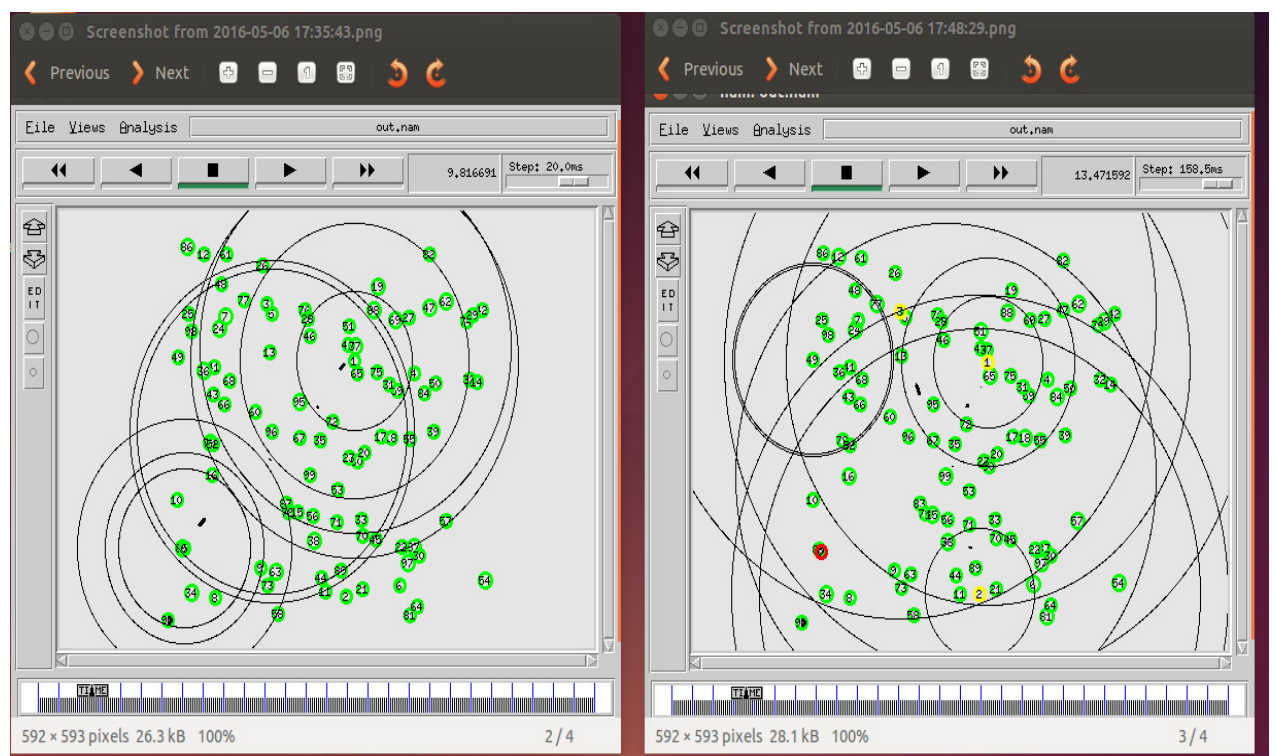

Figure 4. Detection of Minimal Bottleneck and Bottleneck Nodes in NS2

\begin{tabular}{|c|c|c|c|}
\cline { 2 - 4 } \multicolumn{1}{c|}{} & $\begin{array}{c}\text { Average Network } \\
\text { Lifetime (sec) }\end{array}$ & $\begin{array}{c}\text { Average Throughput } \\
\text { (kbps) }\end{array}$ & $\begin{array}{c}\text { Average } \\
\text { PDF (\%) }\end{array}$ \\
\hline Present System & 308.546 & 328.98 & 55.69 \\
\hline RandomAdd_thin & 349.762 & 434.31 & 71.35 \\
\hline RandomAdd_entire & 365.350 & 456.71 & 74.53 \\
\hline RandomAdd_target| & 361.956 & 476.86 & 77.50 \\
\hline
\end{tabular}

Table 2. Shows Parameter Computation 
International Journal of Next-Generation Networks (IJNGN) Vol.8, No.2/3, September 2016

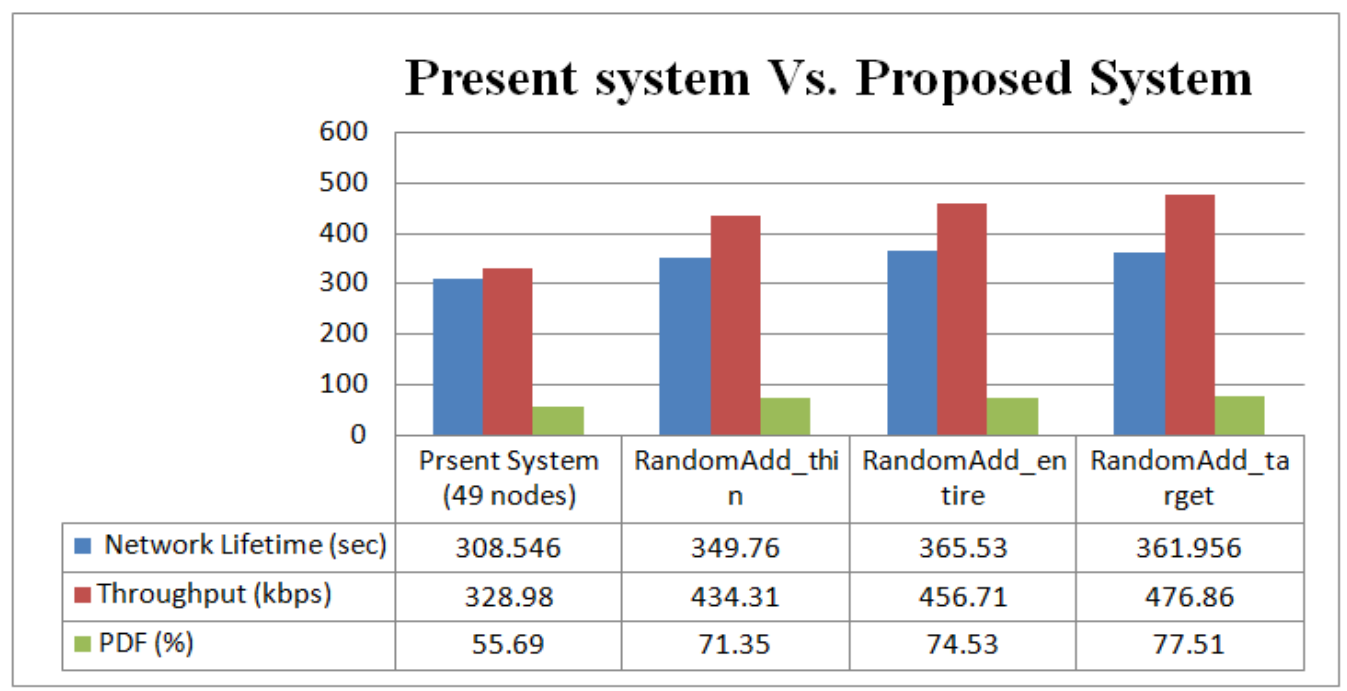

Figure 5. Shows parameter computation in thin, entire, and targeted regions

Figure 5. Depicts comparision between present system and proposed system. Proposed system consists of random additional deployment in thin, entire, and targeted additional deployment strategies.

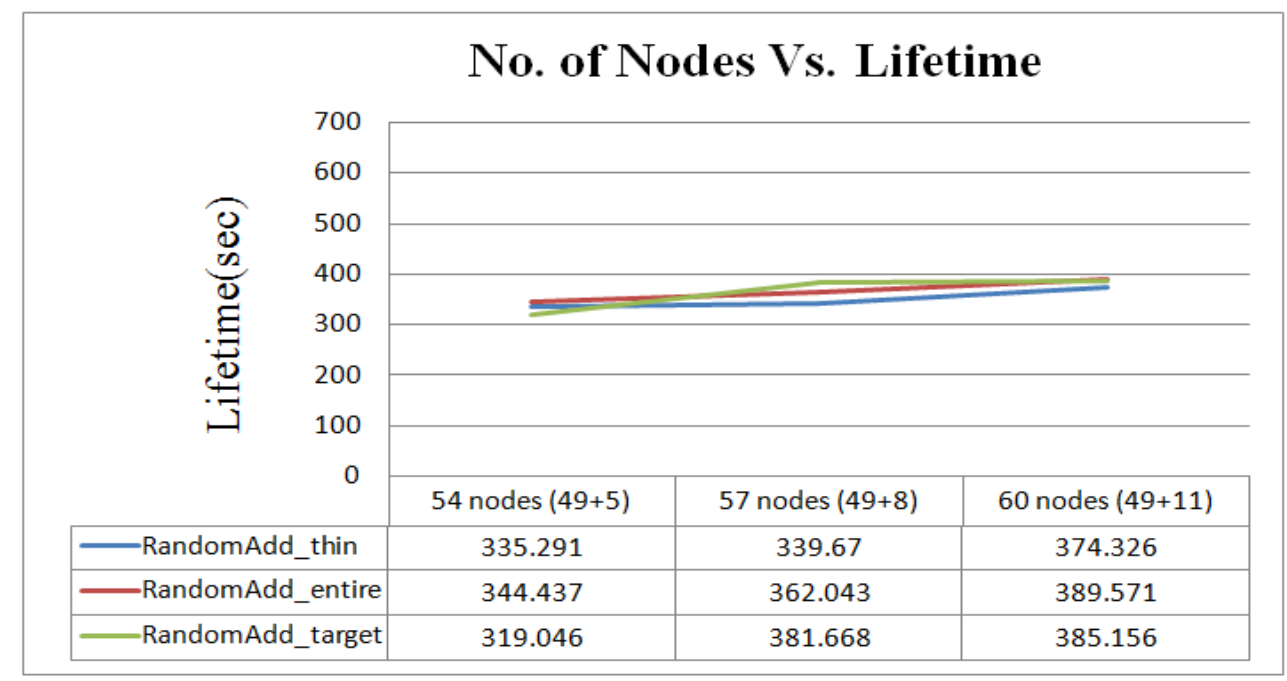

Figure 6. Shows Average Lifetime Computation

Figure 6. Depicts comparision between number of nodes and lifetime in thin, entire and targeted region. Network lifetime is enhanced with increase in number of nodes and gives better results than present system. 


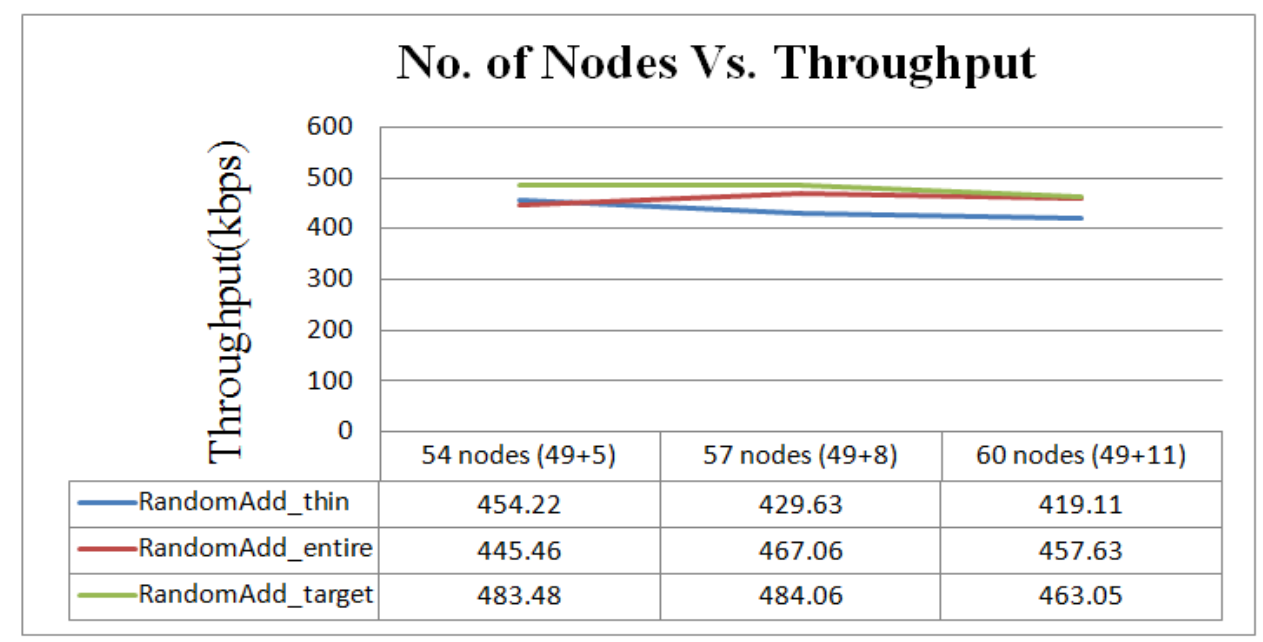

Figure 7. Shows Average Throughput Computation

Figure 7. Depicts comparision between number of nodes and throughput in thin, entire, and targeted region. Proposed work ensures better PDF than that of present system.

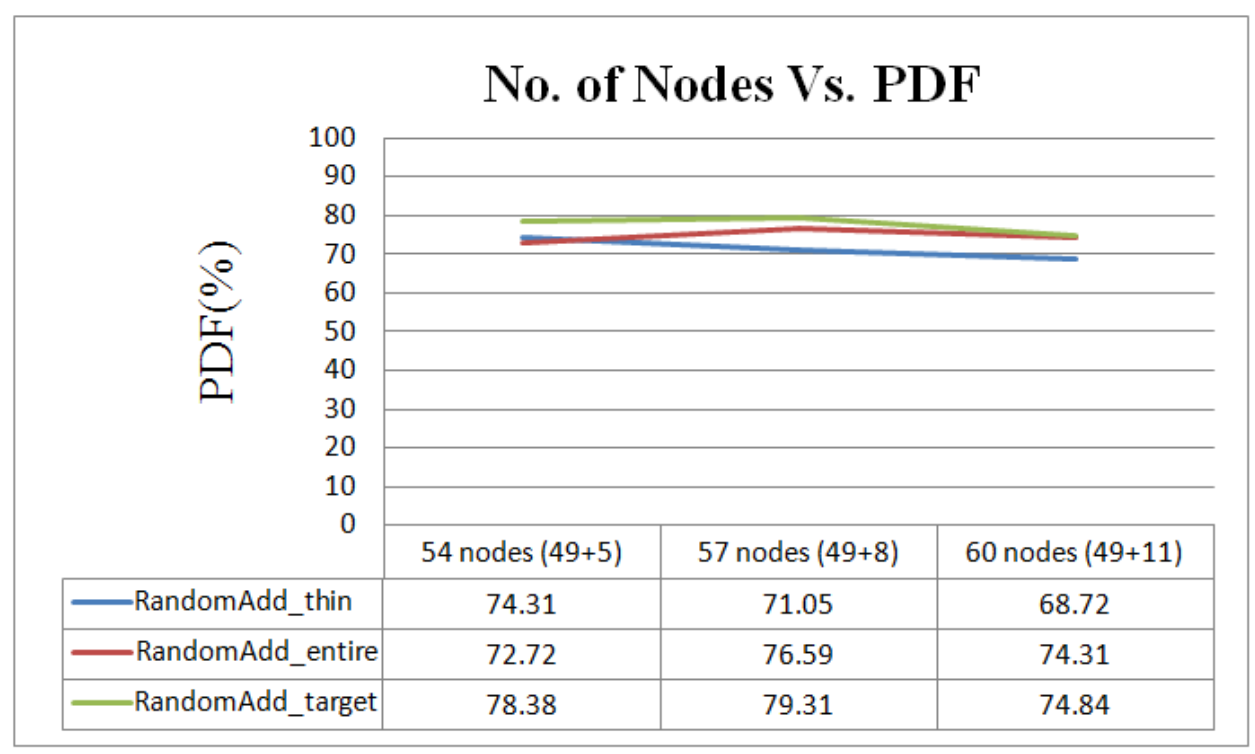

Figure 8. Shows Average PDF Computation

Figure 8. Depicts comparision between number of nodes and packet delivery fraction in thin, entire and targeted region. Proposed work ensures better PDF than that of present system.

\section{CONCLUSION}

The proposed system is based on identification of minimal bottleneck and bottleneck regions in network topology of wireless sensor networks. In simulation work, two additional deployment strategies are used : random and targeted. Random additional sensor deployment is done in thin and entire regions of network topology whereas targeted additional sensor deployment is done in 
targeted areas of network topology. In simulation work parameters like network lifetime, throughput and packet delivery fraction (PDF) are computed to analyze the proposed work. Network lifetime is enhanced with increase in number of nodes. Random additional sensor deployment in thin region achieves $41.2 \%$ increase in lifetime whereas random additional deployment in entire region achieves $56.8 \%$ increase in network lifetime than present system. Targeted additional sensor deployment achieves 53.4\% increase in lifetime. The proposed system achieves maximum throughput than present work. Packet delivery fraction in thin, entire, and targeted regions are $15.6 \%, 18.84 \%, 21.8 \%$ and is maximum than that of present system. Thus, the proposed study achieves energy optimization, by finding the minimal bottleneck and bottleneck regions in network topology and replacing those regions with high energy nodes. Parameters like lifetime, throughput and packet delivery fraction are computed. Simulation results verify the performance of algorithm and gives better results as compared to present system.

\section{FUTURE SCOPE}

Effects of repeated additional sensor node deployment strategies can be studied as a future work. In future work, effects of additional node deployment strategies based on more number of disjoint barriers can be studied. Deployment error and localization error may be considered for more realistic simulations.

\section{REFERENCES}

[1] I. F,Su W Sankarasubramanian Y,Cayirci E Akyildiz, "Wireless Sensor Network A Survey," Computer Networks, Vol. 1, Pp. 393-422, 2002.

[2] He And Shi, "Constructing Sensor Barriers With Minimum Cost In Wsn," Journal Of Parallel Distributed Computing, Pp. 1654-1663, 2012.

[3] Diying Li, Wenping Chen, Qinghua Zhu Huan Ma, "Energy Efficient K-Barrier Coverage In Limited Mobile Wsn," Computer Communications, Pp. 1749-1758, 2012.

[4] Ajay K Sharma Monica, "Comparative Study Of Energy Consumption For Wsn Based On Random And Grid Deploymant Strategies," In International Conference Of Computer Applications, Sept, Pp. 28-35.

[5] O. Dousse,P. Nain And D. Towsley B. Y. Liu, "Dynamic Coverage Of Mobile Sensor Networks," Ieee Trans. Parallel Distri. Syst., Vol. 24, Pp. 301-311, Feb 2013.

[6] G. Yang And D. Qiao, "Multi Round Sensor Deploymant For Guaranteed Barrier Coverage," In Proc. Ieee Infocom, 2010.

[7] K. Wang,H. Liu J. Du, "Maximizing The Lifetime Of K-Discrete Barrier Coverage Using Mobile Sensors," Sensors Journal, Pp. 4690-4701, 2013.

[8] Xiaojun Liu,N. B. Shroff Joohwan Kim, "Minimizing Delay And Maximizing Lifetime For Wsn With Anycast".

[9] L. Narayanan And J. Opatrny M. Eftekhari, "On Multi-Round Sensor Deployment For Barrier Coverage I," Inn Proc. 10th Ieee Mass, 2013.

[10] Chi-Ying Tsui,And Ying Jun Feng Liu, "Joint Routing And Sleep Scheduling For Lifetime Maximization Of Wsn," Ieee, Vol. 9, July 2010.

[11] M. Aida M. Ishizuka, "Performance Study Of Node Placement In Sensor Networks," In In Proc. 24th International Conference On Distributed Computing Systems Workshops, Japan, 2004.

[12] S Kumar And T. H. Lai A. Chen, "Designing Localized Algorithms For Barrier Coverage," In In Proc. Acm Mobicom, 2007.

[13] H. Hassanein,Q. Wang K. Xu, "Relay Node Deploymant Strategies In Heterogeneous Wsn:Single Hop Communication Case," In In Proc. Globecom, 2005.

[14] T. H. Lai,P. Sinha S. Kumar, "Optimal Sleep Wakeup Algorithms For Barriers Of Wireless Sensors," In Proc. Ieeeebroadnets, 2007.

[15] L. R. Ford And D. R. Fulkerson, "Maximal Flow Through A Network," Canadian Journal Of Mathematics, Pp. 399-404. 
International Journal of Next-Generation Networks (IJNGN) Vol.8, No.2/3, September 2016

[16] W. Li And C. G. Cassandras, "A Minimum-Power Wsn Deployment Scheme," In Proc. Ieee Wcnc.

[17] S. Movaghati And M. Ardakani M. Noori, "Characterizing The Path Coverage Of Random Wsn ," Eurasip Journal On Wireless Communications And Networking, Pp. 1-11, 2010.

[18] T. H. Lai And A. Arora S. Kumar, "Barrier Coverage With Wireless Sensors," In In Proceedings Of Acm Mobicom , 2005.

[19] B. Zhang,X. Shen And Z. Yao L Li, "A Study On The Weak Barrier Coverage Problem In Wsn," Computer Networks, Vol. 55, Pp. 711-721, 2011.

[20] D. Qiao G. Yang, "Barrier Information Coverage With Wireless Sensor Networks," In 28th Ieee International Conference On Computer Communications,Inficom, Brazil, 2009, Pp. 918-926.

[21] J. Tang,W. Zhang L Zhang, "Strong Barrier Coverage With Directiional Sesnors," Ieee Global Telecommunications Conference ,Globecom, Pp. 1-6, 2009.

[22] A. Giridhar And P. R. Kumar, "Maximizing The Functional Lifetime Of Sensor Networks," In Proc. 4th Acm Symp. Ipsn, P. 2, 2005.

[23] Y. Bail,H. Ji,J.Ma,Y. Tian J. Li, ""Power: Planning And Deploymant Platform For Wireless Sensor Networks," Fifth International Conference On Grid And Cooperative Computing Workshops Ieee, Pp. 432-436, Oct 2006.

[24] A. Mahajan P. Gajbhije, "A Survey Of Architecture And Node Deployment In Wireless Sensor Networks," Ieee, Pp. 426-430, Aug 2008.

[25] S. Zhang X. Wang, "Comparision Of Several Deployment Strategies In Wireless Sensor Networks," International Conference On E-Health Networking,Digital Ecosystems And Telecommunications, Vol. 1, Pp. 236-239, April 2010.

[26] Gao Jun Fan And Sh Yao Jin, "Coverage Problem In Wsn:A Survey," Journal Of Networks, Vol. 5, Sept 2010.

[27] Zijian Wang And Boleslaw K. Szymanski Eyuphan Bulut, "The Effect Of Neifhbor Graph On Coverage Redundancy In Wsn," In Proc. Of The Ieee Conference On Communications, Pp. 1-5, May 2010.

[28] R. Srikant,N. Shroff S. Shakottai, "Unreliable Sensor Grids:Coverage,Connectivity,And Diameter," Ieee Infocom, Vol. 2, Pp. 1073-1083, 2003.

[29] Y. T. Hou,L. Lai,Y. Shi,S. Shen J. Pan, "Topology Control For Wireless Sensor Networks," Acm Mobicom, Pp. 286-299, 2003.

[30] C. Rosenberg,D. Kofman,N. Shroff V. Mhatre, "A Miminum Cost Heterogeneous Sensor Network Wit Lifetime Constraint," Ieee Transactions On Mobile Computing, 2005.

[31] Paul Meeneghan And Declan Delaney, "An Introduction To Ns,Nam And Otcl Scripting," April 2004.

[32] Dr. M. Sailaya,P. Ramesh,E. Nageswarrarao,V. Rajesh S. Kotes Wararao, "Sensor Networks Simulation In Ns2.26," Iject, Vol. 2, Dec 2011.

\section{AUTHORS:}

Vinaya V. Yarate is a Mtech CSE student in Gogte Institute of Technology, Belgaum, India. Her area of interest is Wireless Sensor Networks.

Dr. Harish H. Kenchannavar is currently working as a Professor in GIT, Belgaum, India. He pursued $\mathrm{PhD}$ degree and has published a number of technical papers in the field of Wireless Sensor Networks.

Prof. Umesh M. Kulkarni is currently working as a Assistant Professor in GIT, Belgaum, India.
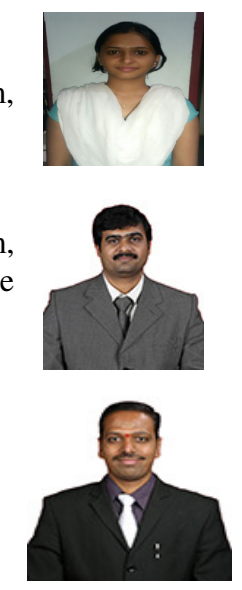